\title{
A Rare Case of Infected Cervicodorsal Intramedullary Epidermoid Cyst
}

\author{
Pawan Chawla ${ }^{1} \quad$ Milind Sankhe $^{1}$ \\ 1Department of Neurosurgery, P. D. Hinduja National Hospital \& \\ Medical Research Centre, Mumbai, Maharashtra, India
}

Address for correspondence Pawan Chawla, DNB, Department of Neurosurgery, P. D. Hinduja National Hospital \& Medical Research Centre, Mumbai, Maharashtra 400016, India (e-mail: ficus_pwn@yahoo.co.in).

Indian J Neurosurg 2021;1:80-84.

Introduction Intramedullary spinal epidermoid cysts have a reported incidence of less than $1 \%$ of intramedullary spinal cord tumors; and out of these, intramedullary epidermoid lesions within the cervical spinal cord are even rarer. Here, we present the case of an adult patient with an infected intramedullary epidermoid cyst, identified on magnetic resonance imaging (MRI) at the C7-D1 level that was successfully excised without any worsening of neurological status.

Case Presentation A 23-year-old female patient presented with history of moderate degree intermittent fever followed by gradually progressive right-sided weakness. She had paresthesia below $\mathrm{C} 5$ dermatome on the right side. On close inspection of the back, a tiny sinus opening was noted at the D2 level near the midline. MRI of the spine showed an intramedullary lesion at the C6-D1 level with the tract connecting to the subcutaneous lesion. Somatosensory evoked potential (SSEP) monitoring was done during the surgery with near total excision of the lesion.

Conclusion Even though the location is rare, a differential diagnosis of the epidermoid should always be considered. A tiny dermal sinus opening helped us in proper diagnosis, along with surgical excision, by providing us a direct path to the intramedullary lesion. An early diagnosis and treatment with neurophysiological monitoring helped us in the maximum safe resection of the lesion without developing any new deficit.

\section{Introduction}

Epidermoid cysts arise from the ectopic entrapment of ectodermal tissue remnants during the 3rd and 5th week of gestation and during neural tube closure. Intramedullary spinal epidermoid cysts have a reported incidence of less than $1 \%$ of intramedullary spinal cord tumors and out of these, intramedullary epidermoid lesions within the cervical spinal cord are even rarer. ${ }^{1-3}$ Dermal sinus tract can be associated with the inclusion tumors (dermoid, epidermoid, and teratoma) in up to $60 \%$ of patients. ${ }^{3,4}$ Sinus tracts are very rarely seen in cervical region as compared with the lumbar and sacral regions. Although these are benign lesions, they can still lead to severe neurological deficits; hence, if identified, these

DOI https://doi.org/ $10.1055 / \mathrm{s}-0040-1708079$ ISSN 2277-954X. lesions are sometimes removed prophylactically to avoid infections and neurological deficits.

Here, we present the case of an adult patient with an infected intramedullary epidermoid cyst, identified on magnetic resonance imaging (MRI) at the C7-D1 level that was successfully excised without any worsening of neurological status.

\section{Case Report}

A 23-year-old female patient presented with a history of moderate degree intermittent fever since the past 15 days, followed by insidious onset, gradually progressive, rightsided weakness since the past 12 days in the form of difficulty

(C) 2020. Neurological Surgeons' Society of India.

This is an open access article published by Thieme under the terms of the Creative Commons Attribution-NonDerivative-NonCommercial-License, permitting copying and reproduction so long as the original work is given appropriate credit. Contents may not be used for commercial purposes, or adapted, remixed, transformed or built upon. (https://creativecommons.org/licenses/by-nc-nd/4.0/)

Thieme Medical and Scientific Publishers Pvt. Ltd., A-12, 2nd Floor, Sector 2, Noida-201301 UP, India 
in holding objects in the right hand and difficulty in wearing sandals on the right foot, leading to with slipping.

She was also complaining of numbness and stiffness of the right upper and lower limb, involving the right side of the chest and trunk. She experienced neck stiffness. She was started on antibiotics at a local hospital and later referred to us for further management. There was no history of trauma or any procedure on the spine.

On neurological examination, she had power of grade $4+/ 5$ in the right lower limb with weakness in the right-hand grip with right pronator drift. She had paresthesia below C5 dermatome on the right side. On close inspection of back, a tiny sinus opening was noted at the D2 level near midline (-Fig. 1).

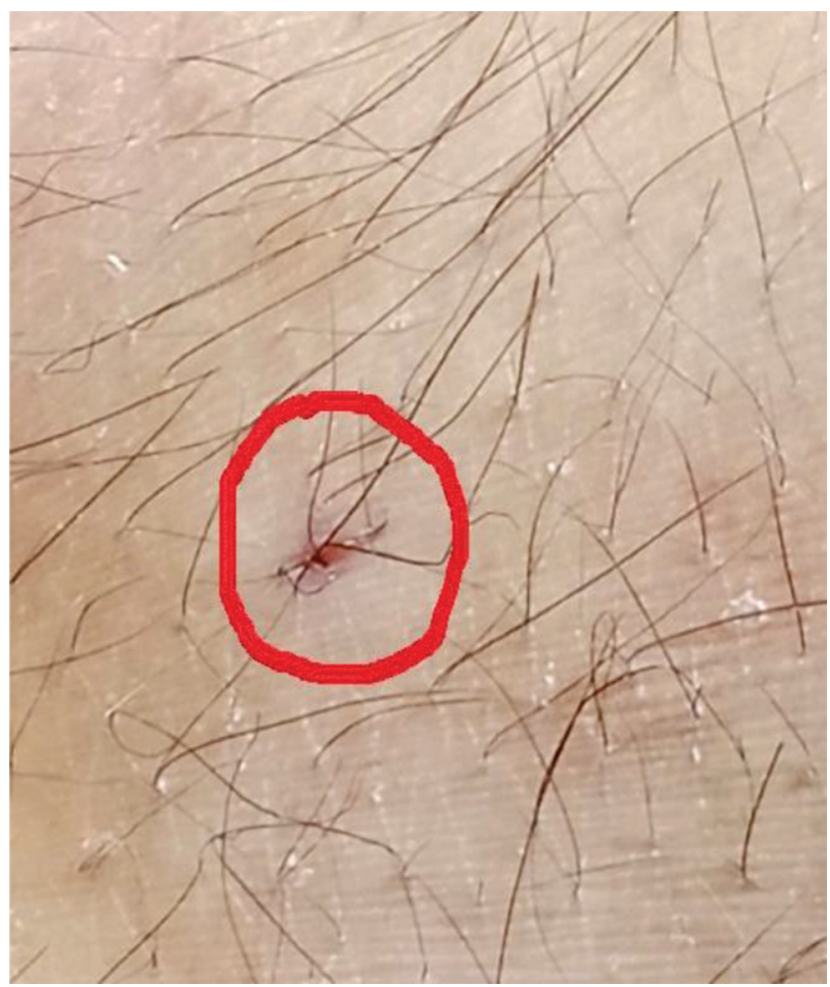

Fig. 1 A tiny dermal sinus opening at the D2 level near the midline.
On evaluation, MRI spine shows an intramedullary, multilocular, variable-sized lesion at the C6-D1 level with hypointense on $\mathrm{T} 1$, hyperintense on $\mathrm{T} 2$ with nodular contrast enhancement at the C7-D1 level, with perilesional edema extending from C4 to D3 with cord expansion and obliteration of surrounding subarachnoid space ( - Fig. 2 ).

The posterior subcutaneous fat at the D1-D2 vertebrae level shows midline, ill defined, irregular-shaped, low signal on T1/T2 sequences with intense nonuniform contrast enhancement, suggesting connection between the spinal cord lesion through the posterior neural arch bony defect. Congenital fusion of C5, C6, C7 and D3, D4, D5 vertebrae is seen (-Fig. 3).

During surgery, a tract was noted going from the dermal sinus toward the spinal cord through the spinous process. Pus was seen coming out from the tract which was sent for culture. Tract was followed and the patient underwent C7-D1 laminectomy. Midline durotomy was done. The cord was widened. Lesion was seen continuing inside the cord parenchyma, following a midline dorsal myelotomy; a pearly white and flaky lesion within the cord substance was seen (-Fig. 4). Near total excision of the lesion was performed using microneurosurgical techniques. Small portions of the capsule densely adherent to the cord parenchyma were left behind. Somatosensory evoked potential (SSEP) monitoring was done during the surgery. SSEP was absent on the right side preoperatively and no drop in SSEP was noted on the left side during the surgery

She was empirically started on tablet Linezolid $600 \mathrm{mg}$ twice a day and tablet Ciprofloxacin $500 \mathrm{mg}$ twice a day for 2 weeks. However, the culture growth was negative. Histopathology was suggestive of an epidermoid cyst lined by a keratinized, stratified squamous lining epithelium with granular layer ( - Fig. 5). There was a sinus tract in the subcutaneous tissue, lined by foamy macrophages, lymphocytes, and foreign body type of giant cells.

The patient gradually improved after the surgery and she had power of grade $4+/ 5$ with mild spasticity and was able to walk without support. Follow-up MRI was done after 3 weeks and 3 months of surgery, which showed no visualization of previously observed lesion with regression of cord

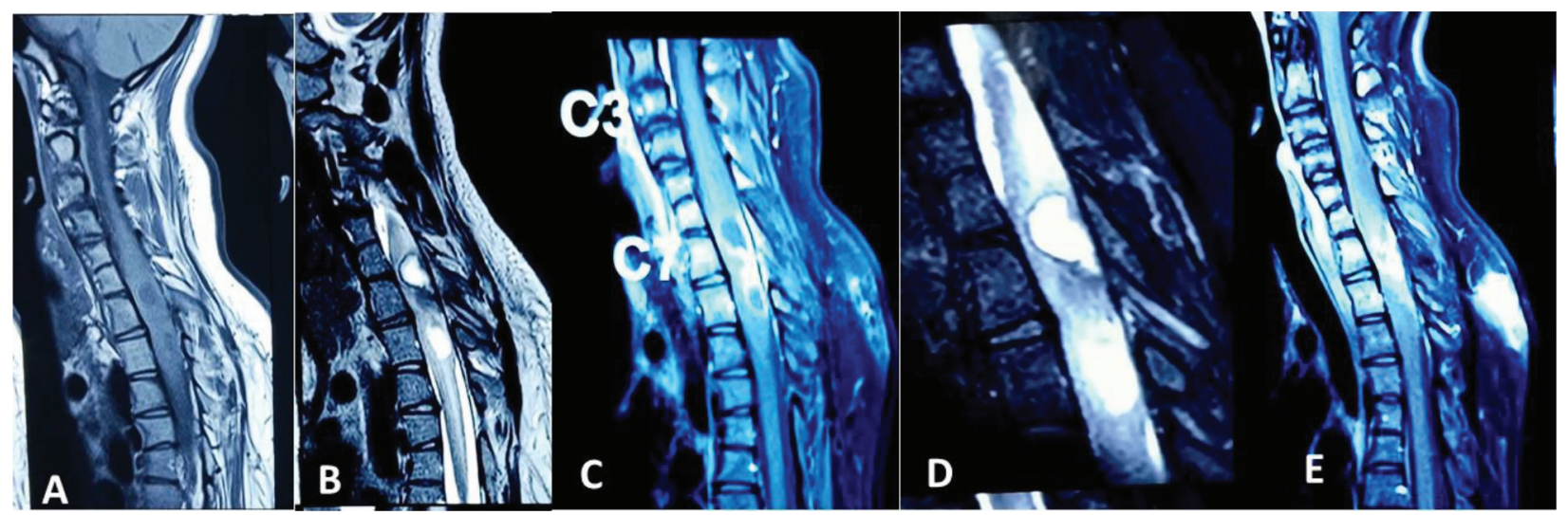

Fig. 2 MRI cervicodorsal spine sagittal imaging (A) T1 sequence; (B, D) T2 sequences; (C, E) postcontrast sequences show intramedullary cervicodorsal lesion with the tract connecting to the subcutaneous lesion. MRI, magnetic resonance imaging. 


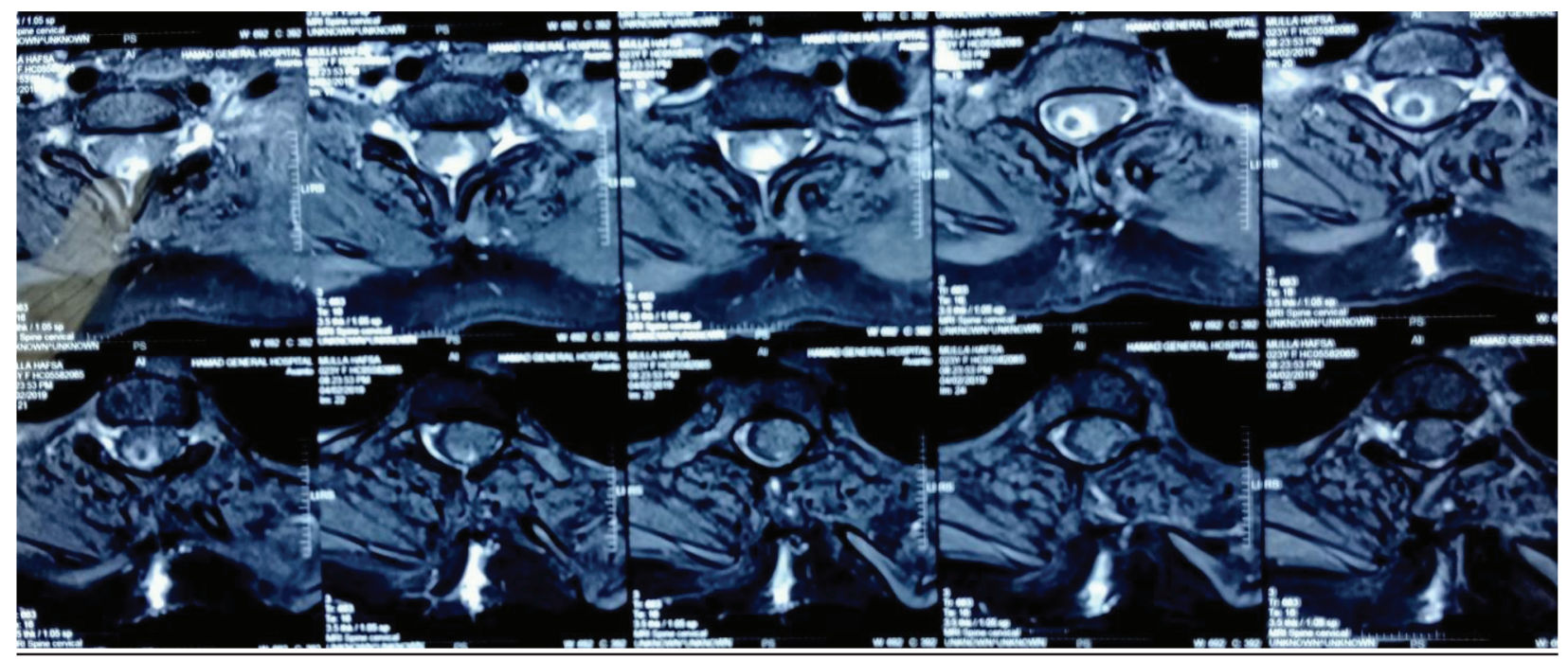

Fig. 3 Axial, postcontrast sequences show a predominantly right-sided intramedullary lesion with enhancement of sinus tract.
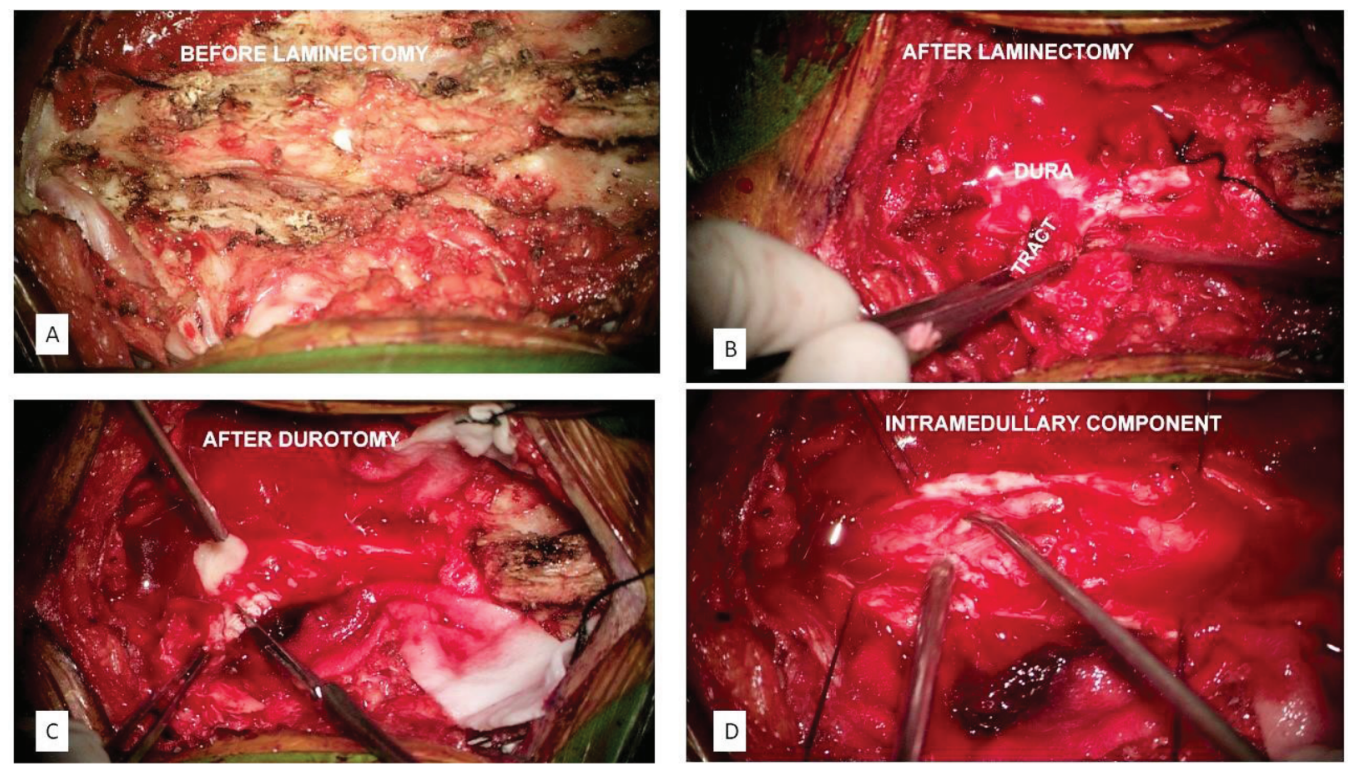

Fig. 4 Intraoperative images. (A) White cheesy material (pus) coming out from the defective spinous process before laminectomy (B). After laminectomy, lesion seen continuing inside the dura mater. (C) Pus seen coming out during excision of extradural component. (D) After durotomy, pus seen continuing inside the cord parenchyma.
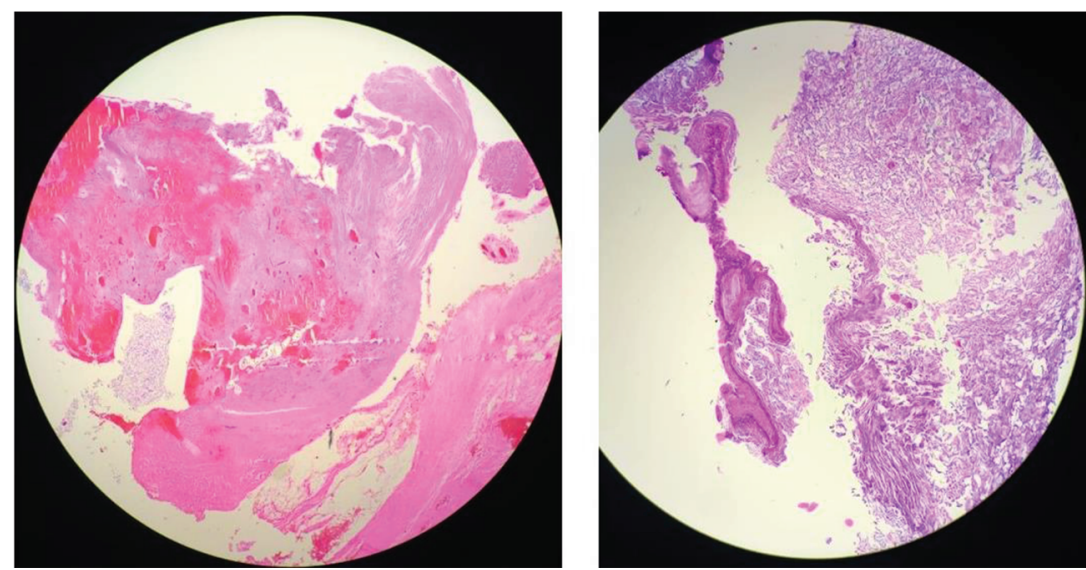

Fig. 5 Histopathology was suggestive of an epidermoid cyst, lined by keratinized stratified squamous lining epithelium with granular layer. 


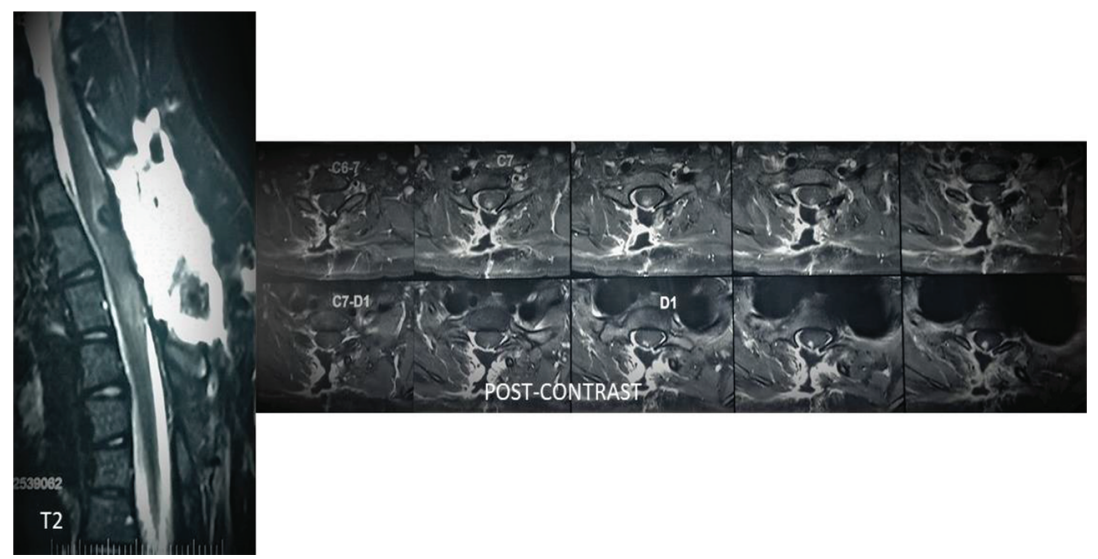

Fig. 6 Postoperative images (3 weeks): T2-weighted sagittal images and postcontrast axial image.

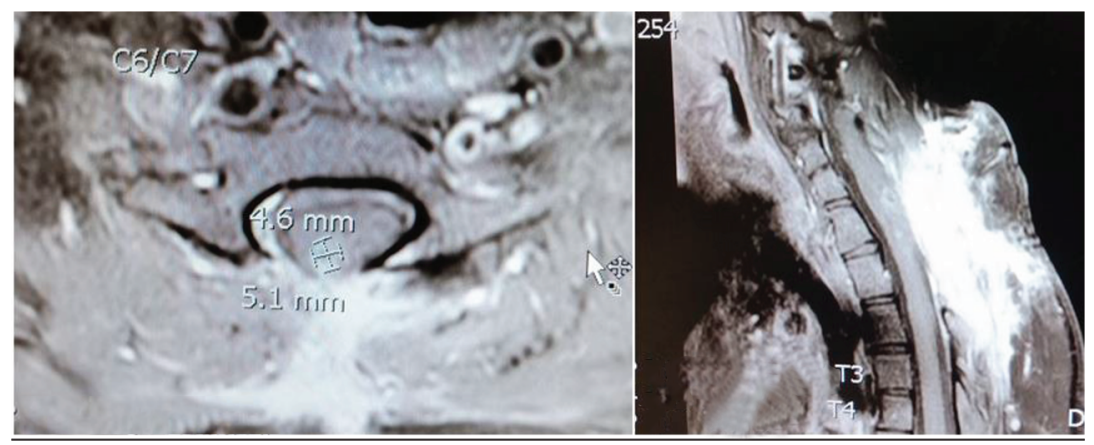

Fig. 7 Postoperative images (3 months): postcontrast axial and sagittal images.

edema. Some linear enhancement was seen at the site of the lesion at the D1-2 level ( - Figs. 6 and $\mathbf{7}$ ).

\section{Discussion}

Most intraspinal epidermoid cysts are subdural and extramedullary, and they rarely develop in the intramedullary compartment. ${ }^{5,6}$ An intramedullary epidermoid cyst is composed of stratified squamous epithelium rarely and there are very few cases ( $<80$ cases) reported in the literature. ${ }^{1}$ These lesions can be either congenital or acquired (might be due to lumbar punctures). ${ }^{1}$

Guidetti and Gagliardi reported a series of 3844 intracranial and 452 intraspinal tumors in patients of all ages. There was an incidence of $0.8 \%$ for cranial and $0.7 \%$ for spinal epidermoid cysts. The incidence of intramedullary epidermoid cysts is still rarer. ${ }^{7}$ Chiari reported the first case in $1833 .^{8,9}$ The thoracic region is the most common site of the intramedullary epidermoid cysts (usually between D4-D8 levels).

Signal intensity patterns are variable, ranging from hypointense to hyperintense, and the tumors are often multiloculated. Typically, the tumor signal is heterogeneous and most often shows hypointensity on $\mathrm{T} 1$-weighted images and hyperintensity on T2-weighted images. ${ }^{6} \mathrm{~A}$ rim of high-signal intensity may be visible on proton density studies; some tumors show rim enhancement with gadolinium administration. The differential diagnosis includes the dermoid, arachnoid cyst, and ependymoma. ${ }^{10-13}$
Here, a young female patient presented with subacute presentation of meningitis and cervicodorsal myelopathy with a rare location of intraspinal epidermoid cyst. The possible source of the infection was the dermal sinus tract, which was identified on close local examination. Local examination is always important and it should be given due respect. On radiological evaluation, the patient was diagnosed to have an intramedullary lesion with a tract connecting it to the dermal sinus. Other bony deformities were noted in the form of failure of segmentation, leading to fusion of various vertebrae and indicating the congenital origin of the lesion.

Culture growth was negative but that can be attributed to the antibiotics that were taken at a local hospital before being referred to us. The patient was empirically started on tablet Linezolid and tablet ciprofloxacin. Even though the location is rare, a differential diagnosis of epidermoid should always be considered. Neurophysiological monitoring help in the maximum safe resection of the lesion without developing any new deficit.

Treatment of spinal epidermoid cyst is surgical. However, if it is tightly adhered to neural structures, it is advisable to leave the adherent cyst capsule to avoid unnecessary neurologic deficits.

\section{Conclusion}

A tiny dermal sinus opening helped us in proper diagnosis, along with surgical excision, by providing us a direct path 
to the intramedullary lesion. Local examination is always important and it should be given due respect.

An early diagnosis and treatment with adjunct neurophysiological monitoring helped us in managing this lesion without developing any further neurological deficit.

\section{Conflict of Interest}

None declared.

\section{Reference}

1 Ogden AT, Khandji AG, McCormick PC, Kaiser MG. Intramedullary inclusion cysts of the cervicothoracic junction. Report of two cases in adults and review of the literature. J Neurosurg Spine 2007;7(2):236-242

2 Tekkök IH. Intramedullary epidermoid cysts. J Neurosurg Spine 2008;8(2):202-203, author reply 203

3 Babayev R, Abbasov B, Ekşi MS. Thoracic intramedullary epidermoid cyst-timely fashion diagnosis and treatment. Childs Nerv Syst 2015;31(5):793-796

4 Bailey IC. Dermoid tumors of the spinal cord. J Neurosurg 1970;33(6):676-681

5 Altman RS. Dermoid tumor of the posterior fossa associated with congenital dermal sinus. Report of a case and review of the literature. J Pediatr 1963;62:565-570
6 Roux A, Mercier C, Larbrisseau A. Dube LJ, Dupuis C, Del Carpio R. Intramedullary epidermoid cysts of the spinal cord. Case report. J Neurosurg 1992;76(3):528-533

7 Graillon T, Rakotozanany P, Meyer M, Dufour H, Fuentes S. Intramedullary epidermoid cysts in adults: case report and updated literature review. Neurochirurgie 2017; 63(2):99-102

8 Guidetti B, Gagliardi FM. Epidermoid and dermoid cysts. Clinical evaluation and late surgical results. J Neurosurg 1977;47(1):12-18

9 Penisson-Besnier I, Guy G, Gandon Y. Intramedullary epidermoid cyst evaluated by computed tomographic scan and magnetic resonance imaging: case report. Neurosurgery 1989;25(6):955-959

10 Osborn AG, Preece MT. Intracranial cysts: radiologicpathologic correlation and imaging approach. Radiology 2006;239(3):650-664

11 Kukreja K, Manzano G, Ragheb J, Medina LS. Differentiation between pediatric spinal arachnoid and epidermoid-dermoid cysts: is diffusion-weighted MRI useful? Pediatr Radiol 2007;37(6):556-560

12 Bloomer CW, Ackerman A, Bhatia RG. Imaging for spine tumors and new applications. Top MagnReson Imaging 2006;17(2):69-87

13 Teksam M, Casey SO, Michel E, Benson M, Truwit CL. Intraspinal epidermoid cyst: diffusion-weighted MRI. Neuroradiology 2001;43(7):572-574 